\title{
Of SMN in mice and men: a therapeutic opportunity
}

\author{
Kathryn J. Swoboda
}

Department of Neurology, University of Utah, Salt Lake City, Utah, USA.

\begin{abstract}
Spinal muscular atrophy (SMA) is an autosomal recessive neurodegenerative disease that predominantly affects motor neurons, resulting in progressive muscular atrophy and weakness. SMA arises due to insufficient survival motor neuron (SMN) protein levels as a result of homozygous disruption of the SMN1 gene. SMN upregulation is a promising and potent treatment strategy for this currently incurable condition. In this issue of the JCI, two independent research groups report novel observations in mouse models of severe SMA that provide hope that this approach will afford meaningful benefit to individuals with SMA.
\end{abstract}

Spinal muscular atrophy (SMA) is a frequently fatal autosomal recessive neurodegenerative disorder characterized by motor neuron cell loss in the anterior horns of the spinal cord and brain stem (Figure 1). SMA results in skeletal muscle weakness, atrophy, and premature death in the majority of those affected. With an incidence of 1 in 10,000 births, it is the most commonly inherited motor neuron disease and a leading genetic cause of mortality in infants and children (1). Since its original descriptions by Werdnig and Hoffman independently in the 1890s (2, 3), an increasingly broad spectrum of SMA phenotypes has been identified, ranging from prenatal to adult onset (Table 1). SMA type I, the severe infantile form, accounts for more than $50 \%$ of newly diagnosed cases. Infants with this condition manifest hypotonia and weakness before six months of age. An effective therapeutic for SMA has thus far remained elusive. Treatment currently involves managing symptoms and preventing complications. However, the unique genetic and clinical characteristics of SMA make it one of the most promising candidates for the development of an effective and potentially curative therapeutic intervention.

\section{A tale of two SMNs}

More than $95 \%$ of individuals with SMA possess a homozygous deletion involving exon 7 of their survival motor neuron 1 (SMN1) gene $(4,5)$. Thus, the majority of

Conflict of interest: The author has declared that no conflict of interest exists.

Citation for this article: J Clin Invest. 2011; 121(8):2978-2981. doi:10.1172/JCI58752.
SMA patients, regardless of phenotype, share a unique genetic signature that is easily identified via a simple and inexpensive DNA test $(6,7)$. While homozygous SMN1 deletion is necessary, it is insufficient to cause SMA unless SMN2 is present. An inverted duplication of chromosome $5 \mathrm{q} 13$ unique to a subset of hominids including humans harbors up to several copies of SMN1 and its nearly identical twin, SMN2 $(8,9)$. A critical nucleotide difference in SMN2 results in the exclusion of exon 7 from the majority of transcripts yet produces a fraction of the identical full-length SMN protein as SMN1 (ref. 10 and Figure 2). Thus, the presence of SMN2 rescues the embryonic lethality observed in other species with homozygous SMN1 deletion, testament to the critical role of SMN in the spliceosomal assembly of small nuclear ribonucleoproteins (snRNPs) (11).

The opportunity to target SMN2 gene(s) or transcripts in vivo to rescue levels of SMN protein in patients with SMA has been widely viewed as an unprecedented opportunity for therapeutic success (12). Proof of concept for this strategy is based on several compelling observations replicated in numerous laboratories over the past decade. These include the successful targeting of SMN2 in vitro in cells from human subjects (13-15); the repeated demonstration of successful upregulation, replacement, or repair of SMN2 in vivo in animal models of SMA and in human subjects (16-20); and the observation that increasing SMN2 dosage in a severely affected mouse model is sufficient to rescue the phenotype (21). Finally, a modifying impact of SMN2 dosage on disease pheno- type in SMA human populations has been repeatedly demonstrated, with infants with SMA type I much more likely to have two or fewer SMN2 copies as compared to those with milder phenotypes $(22,23)$. In this issue of the JCI, two independent groups of researchers address critical issues that continue to provide uncertainty as we try to translate observations from mouse models to the clinic: in particular, these include the relevance of certain recent observations in severe mouse models, such as cardiomyopathy, and the degree to which already symptomatic patients, particularly those with SMA type I, are likely to benefit from SNM2 targeting $(24,25)$.

\section{Prolactin: another therapeutic candidate?}

In this issue, Farooq and colleagues demonstrate that the delivery of a novel therapy, prolactin (PRL), increases SMN expression in both mouse models of SMA and human cell lines via a transcriptional mechanism (24). In aggregate, their in vitro data indicate that the effect of PRL on SMN is almost certainly via the STAT5 pathway. STAT5 is a member of the STAT family of transcription factors that are direct targets of JAKs, which are activated, in turn, by ligand binding via specific cytokine receptors. Prolactin is a small polypeptide protein that binds to PRL receptors triggering the JAK2/STAT5 signaling pathway. In the animal model of severe SMA, the ability of subcutaneously delivered PRL to upregulate SMN in brain and spinal cord resulted in a marked improvement in motor function, but only a modest improvement in survival (24). What makes this story potentially more compelling than that of other candidates with similar capacity for SMN upregulation is that this effect may prove more robust in humans than in mice because of the presence of a higher number of STAT5-binding sites in the human SMN2 promoter. In addition, PRL crosses the blood-brain barrier, and since recombinant PRL has been previously tested and proven safe in mothers of preterm infants 


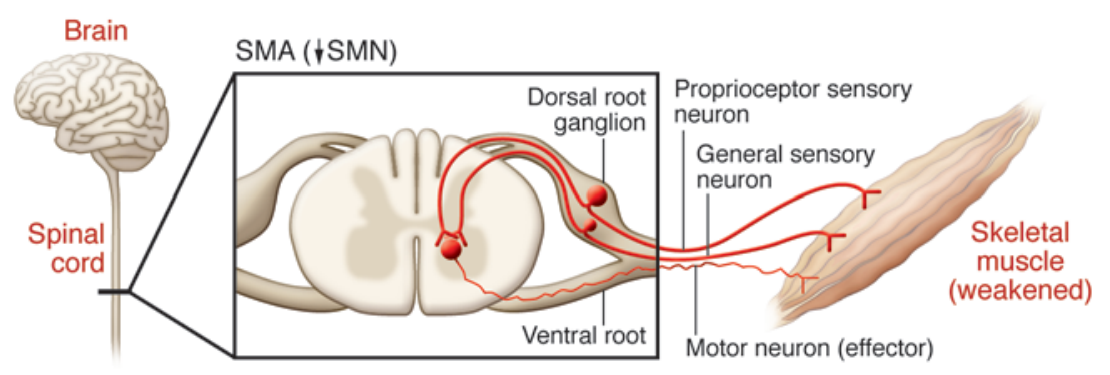

to augment lactation, the immediate potential therapeutic implications are evident as we await other SMN-targeted therapies to navigate the hurdles of the clinical trials pipeline (26). As such, PRL joins a short list of candidates potentially worthy of further testing in SMA patients in the near term.

\section{Inducing SMN in SMA mice: a postsymptomatic rescue}

Since patients present at various stages of disease progression and SMN restoration therapy in individuals with SMA would occur after symptom onset, a key question is the following: When is SMN upregulation or replacement too late, and when is it "just in time"? To try to answer this question, Lutz and colleagues generated SMA model mice harboring an inducible Smn rescue allele (25). Inducing expression of SMN to an estimated $40 \%$ of wild-type levels following onset of symptoms at P4 in an animal model of severe SMA resulted in a profound improvement in motor function and survival. While induction at P4 clearly and dramatically improved survival, that at P6 and later time points resulted in a much more modest impact on survival and motor function that was entirely absent by P10. In mice normally expected to die by $\mathrm{P} 17$, this is a compelling achievement, but what does it really mean for patients with SMA? At P4, motor neuron cell counts in spinal cord in affected mice are equivalent

\section{Figure 1}

SMA is a neurodegenerative disease that predominantly affects motor neurons. SMA is caused by insufficient levels of SMN protein as a result of homozygous disruption of the SMN1 gene. The primary target of SMN deficiency appears to be a relatively selective degeneration of motor neurons in the anterior horn cells of the brainstem and spinal cord, resulting in skeletal muscle atrophy.

to those in wild-type mice. This observation, along with functional studies in mice demonstrating impaired synaptic vesicle release at presynaptic neuromuscular junctions (NMJs), have led investigators to postulate that motor neuron cell loss is a rather late event in SMA disease pathogenesis (27). However, in contrast to patients, these symptomatic mice, untreated until $\mathrm{P} 4$, have much more subtle evidence of denervation and certainly more preserved motor function as compared with symptomatic SMA type I infants. Thus, exactly how well the observations of Lutz and colleagues (25) will translate to patients with SMA, at least to those with the severe infantile form, is not entirely clear.

Table 1

Spectrum of phenotypic manifestations in proximal SMA

\begin{tabular}{|c|c|c|c|c|}
\hline \multirow[t]{2}{*}{ SMA type } & Typical age of onset & Typical life span & Also called & Clinical characteristics \\
\hline & & & & Maximum milestones achieved \\
\hline \multirow[t]{2}{*}{0} & Prenatal & $<6$ months & $\begin{array}{c}\text { SMA - arthrogryposis multiplex } \\
\text { congenita type }\end{array}$ & $\begin{array}{l}\text { Congenital hypotonia, weakness, respiratory } \\
\text { failure, proximal joint contractures }\end{array}$ \\
\hline & & & & Unable to breathe unsupported \\
\hline \multirow[t]{2}{*}{ I } & Birth-6 months & $\begin{array}{l}\sim 32 \% \text { survival } \\
\text { probability }>2 \text { years }{ }^{A}\end{array}$ & Werdnig-Hoffman disease & $\begin{array}{l}\text { Infantile onset of generalized hypotonia, } \\
\text { weakness, impaired bulbar function, } \\
\text { respiratory insufficiency }\end{array}$ \\
\hline & & & & Unable to sit unsupported \\
\hline \multirow[t]{3}{*}{ II } & 6-12 months & $\sim 70 \%$ survival to adulthood ${ }^{A}$ & SMA, Dubowitz type & Able to sit independently \\
\hline & & & & $\begin{array}{l}\text { Onset of limb weakness } \\
\text { as infants or toddlers }\end{array}$ \\
\hline & & & & $\begin{array}{l}\text { Progressive weakness, respiratory } \\
\text { insufficiency, scoliosis, joint } \\
\text { contractures in childhood }\end{array}$ \\
\hline Illa & After 12 months & Normal & Kugelberg-Welander disease & $\begin{array}{l}\text { Onset of proximal muscle weakness } \\
\text { in childhood }\end{array}$ \\
\hline IIIb & After 3 years & & Kugelberg-Welander disease & $\begin{array}{l}\text { Able to ambulate independently, although } \\
50 \% \text { with type IIla lose independent } \\
\text { ambulation by } 12 \text { years of age }\end{array}$ \\
\hline IV & Adulthood & Normal & & $\begin{array}{l}\text { Onset of proximal leg weakness in } \\
\text { adulthood, able to ambulate independently }\end{array}$ \\
\hline
\end{tabular}

AData from ref. 29. 


\section{Chromosome $5 q$}

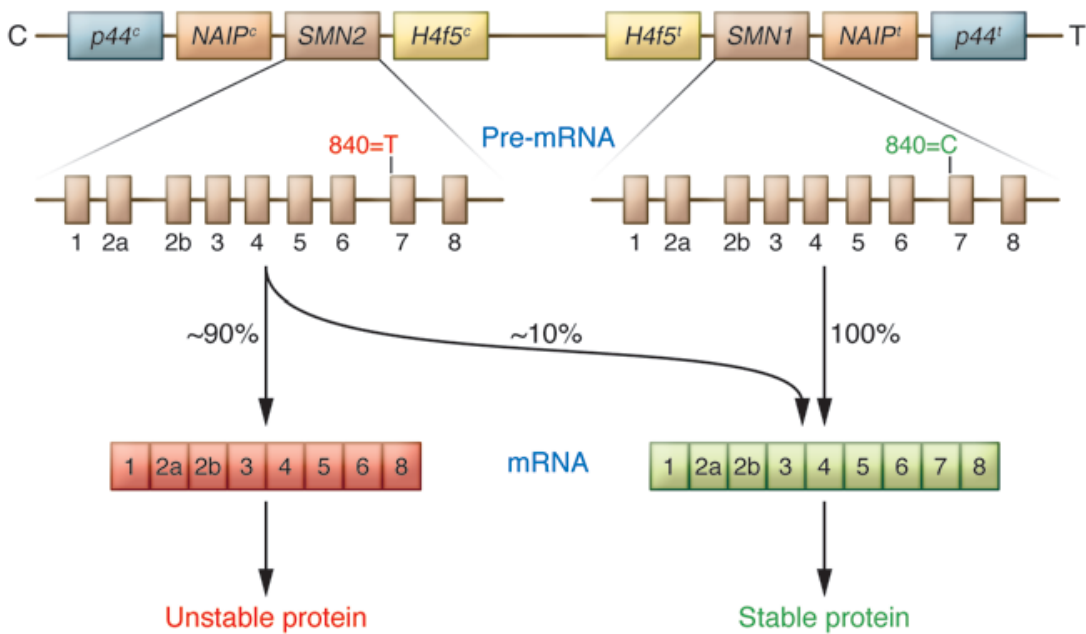

\section{Figure 2}

Gene organization and expression in the region of human chromosome $5 q$ containing the SMN1 and SMN2 genes. SMN is one of four duplicated genes within a duplicated inverted 500-bp region on chromosome $5 q$. The two copies of SMN differ by a few base pairs with only two of them being in the exonic sequence. Functionally critical is the single nucleotide difference in exon 7 at position 840. Although translationally silent, the $\mathrm{C} \rightarrow \mathrm{T}$ transition in SMN2 affects splicing. SMN is one of four genes in this inverted duplicated region; the others have not been clearly demonstrated to contribute to the pathogenesis of SMA. H4F5, protein 4F5; NAIP, NLR family, apoptosis inhibitory protein.
Parallel studies of NMJ pathology, muscle denervation, spinal cord pathology, and motor function aren't feasible in human subjects, although such studies have been beautifully performed in mice by Lutz and colleagues (25), and others. However, observations in prospectively evaluated infants with a positive family history and documented homozygous SMN1 exon 7 deletion suggest that distal denervation is indeed a sentinel event in infants at a time when other symptoms of the disease are relatively modest, in contrast with the mouse model data (28). The early and rapid progression of distal denervation in the first few weeks to months of life in infants predicted to develop type I SMA represents perhaps the single greatest challenge to successfully targeting SMN in humans. Infants destined to develop SMA type I unfortunately account for the largest proportion of those born at risk for SMA, highlighting the urgency for prospective identification of such cases via newborn screening to better assess the potential benefit of earlier diagnosis and intervention on improving outcomes for a majority of those who will develop SMA.

\section{Translating lessons from mice to humans}

Whether the mouse models of severe SMA currently in use are robust surrogates for the severe infantile form of the human disease across the spectrum of manifestations observed in the mouse model remains a point of controversy; nevertheless, the increasing number of successful therapeutic interventions targeting SMN in such severe mouse models promise great hope for the development of an effective therapy for SMA. The dramatic nature of the rescue after symptom onset achieved by Lutz et al. (25) provides encouragement that reversing disease pathology may be feasible in patients with SMA. As such, it represents another significant milestone in our quest for demonstrating proof of concept for a meaningful therapeutic benefit with targeted upregulation of SMN. For clinicians who have closely followed developments at the bench to glean insights to their translational relevance, the already demonstrated, although less dramatic, survival benefit observed with interventions targeting increased SMN expression after symptom onset are also compelling. Avila et al. previously demonstrated that treatment with trichostatin A at P5 in the mouse model of severe SMA resulted in a modest impact on survival and motor function (18); likewise, the administration of scAAV9-SMN by Foust and colleagues at P5 demonstrated a modest effect (19). What sets the publication by Lutz and colleagues (25) apart is the dramatic nature of the rescue, heretofore previously matched only by SMN replacement at P1 or P2 $(22,23)$. Thus, the weight of the evidence to date, fortified by the work of Lutz and colleagues (25), suggests that all is not lost after symptom onset in SMA.

Translating the work of Farooq and colleagues (24) from mouse to human is perhaps even more challenging, due to the substantial differences identified between the PRL promoter in mice and humans. However, if the authors prove correct in their interpretation that PRL may prove substantially more potent in upregulat- ing SMN2 in vivo in humans than it did in mice, then it may indeed prove a promising intervention in patients with SMA, even for those already exhibiting symptoms.

Predicting value for a given therapy is profoundly more difficult in patients than in animal models and analogous in some respects to peering into a crystal ball. As we look to the future, we should avoid the temptation to squander the opportunity to achieve modest yet meaningful benefit in already symptomatic patients. The substantial progress of the past decade, particularly the dramatic benefits achieved with a variety of therapeutic strategies targeting SMN in animal models, provides us with an increasing likelihood that at least one of the current therapies under development will prove successful in the next few years. The wealth of data continues to support a much greater benefit for mice treated as early as possible in the course of the disease. This exposes a painful truth that is hard for those of us who are both clinicians and clinical trialists to swallow. In order to have the best chance for a "cure," or even definitive proof of a more modest benefit, we may have to target our early efforts to those with less severe disease, early in the clinical course. For that reason, a broader adoption of newborn screening for SMA may prove the most rapid and effective means of identifying a cohort of infants and children who represent the best candidates for promising therapeutic treatments in the near term. Ultimately this will benefit the greatest number of patients by allowing early qualification of promising therapies. 


\section{Acknowledgments}

This work was supported in part by NIH grants NICHD R01-HD054599 and R01HD69045, and by grants from FightSMA, Families of SMA, and the Muscular Dystrophy Association.

Address correspondence to: Kathryn J. Swoboda, Pediatric Motor Disorders Research Program, Department of Neurology, 30 N. 1900 E. SOM3R149, Salt Lake City, Utah 84132, USA. Phone: 801.585.9717; Fax: 801.587.9346; E-mail: swoboda@genetics.utah.edu.

1. Prior TW, et al. Newborn and carrier screening for spinal muscular atrophy. Am J Med Genet Part A. 2010;152A(7):1608-1616

2. Werdnig G. Zwei Frühinfantile hereditäre Fälle von progressiver Muskelatrophie unter dem Bilde der Dystrophie, aber auf neurotischer Grundlage. Arch Psychiat Nervenkr. 1891;22(2):437-480.

3. Hoffman J. Ueber chronische spinale Muskelatrophie im Kindesalter, auf Familiärer Basis. Beutsch Z Nervenheilk. 1893;3(6):427-470.

4. Lefebvre $S$, et al. Identification and characterization of a spinal muscular atrophy-determining gene. Cell. 1995;80(1):155-165.

5 . Wan $\mathrm{L}$, et al. The survival of motor neurons protein determines the capacity for snRNP assembly: biochemical deficiency in spinal muscular atrophy. Mol Cell Biol. 2005;25(13):5543-5551.

6. Feldkotter M, et al. Quantitative analyses of SMN1 and SMN2 based on real-time lightcycler PCR: fast and highly reliable carrier testing and prediction of severity of spinal muscular atrophy. Am J Hum Genet. 2002;70(2):358-368.
7. Mailman MD, et al. Molecular analysis of spinal muscular atrophy and modification of the phenotype by SMN2. Genet Med. 2002;4(1):20-26.

8. Rochette CF, Gilbert N, Simard LR. SMN gene duplication and the emergence of the SMN2 gene occurred in unique hominids: SMN2 is unique to Homo sapiens. Hum Genet. 2001;108(3):255-266.

9. Monani UR, et al. A single nucleotide difference that alters splicing patterns distinguishes the SMA gene SMN1 from the copy gene SMN2. Hum Mol Genet. 1999;8(7):1177-1183.

10. Lorson CL, Hahnen E, Androphy EJ, Wirth B. A single nucleotide in the SMN gene regulates splicing and is responsible for spinal muscular atrophy. Proc Natl Acad Sci U S A. 1998;96(11):6307-6311.

11. Fischer U, Liu Q, Dreyfuss G. The SMN-SIP1 complex has an essential role in spliceosomal snRNP biogenesis. Cell. 1997;90(6):1023-1029.

12. Kolb SJ, Kissel JT. Spinal muscular atrophy: a timely review [published online ahead of print April 11, 2011]. Arch Neurol. doi:10.1001/archneurol.2011.74.

13. Brichta L, et al. Valproic acid increases the SMN2 protein level: a well-known drug as a potential therapy for spinal muscular atrophy. Hum Mol Genet. 2003;12(19):2481-2489.

14. Andreassi C, et al. Phenylbutyrate increases SMN expression in vitro: relevance for treatment of spinal muscular atrophy. Eur J Hum Genet. 2004; 12(1):59-65.

15. Grzeschik SM, et al. Hydroxyurea enhances SMN2 gene expression in spinal muscular atrophy cells. Ann Neurol. 2005;58(2):194-202.

16 . Brichta $L$, et al. In vivo activation of SMN in spinal muscular atrophy carriers and patients treated with valproate. Ann Neurol. 2006;59(6):970-975.

17. Brahe C, et al. Phenylbutyrate increases SMN gene expression in spinal muscular atrophy patients. Eur J Hum Genet. 2005;13(2):256-259.

18. Avila AM, et al. Trichostatin A increases SMN expression and survival in a mouse model of spinal muscular atrophy. J Clin Invest. 2007;117(3):659-671.
19. Foust KD, et al. Rescue of the spinal muscular atrophy phenotype in a mouse model by early postnatal delivery of SMN. Nat Biotechnol. 2010; 28(3):271-274.

20. Dominguez E, et al. Intravenous scAAV9 delivery of a codon-optimized SMN1 sequence rescues SMA mice. Hum Mol Genet. 2011;20(4):681-693.

21. Monani UR, et al. The human centromeric survival motor neuron gene (SMN2) rescues embryonic lethality in $\operatorname{Smn}(-/-)$ mice and results in a mouse with spinal muscular atrophy. Hum Mol Genet. 2000;9(3):333-339.

22. Wirth B, et al. Mildly affected patients with spinal muscular atrophy are partially protected by an increased SMN2 copy number. Hum Genet. 2006;119(4):422-428.

23. Prior TW, et al. Homozygous SMN1 deletions in unaffected family members and modification of the phenotype by SMN2. Am J Med Genet A. 2004;130A(3):307-310.

24. Farooq F, et al. Prolactin increases SMN expression and survival in a mouse model of severe spinal muscular atrophy via the STAT5 pathway. J Clin Invest. 2011;121(8):3042-3050.

25. Lutz CM, et al. Postsymptomatic restoration of SMN rescues the disease phenotype in a mouse model of severe spinal muscular atrophy. J Clin Invest. 2011;121(8):3029-3041.

26. Powe CE, et al. Recombinant human prolactin for the treatment of lactation insufficiency. Clin Endocrinol (Oxf). 2010;73(5):645-653.

27. Kong L, et al. Impaired synaptic vesicle release and immaturity of neuromuscular junctions in spinal muscular atrophy mice. J Neurosci. 2009; 29(3):842-851.

28. Swoboda KJ, et al. Natural history of denervation in SMA: relation to age, SMN2 copy number and function. Ann Neurol. 2005;57(5):704-712.

29. Zerres K, Wirth B, Rudnik-Schoneborn S. Spinal muscular atrophy-clinical and genetic correlations. Neuromuscul Disord. 1997;7(3):202-207.

\title{
Quantity, not quality, of antibody response decreased in the elderly
}

\author{
Bonnie B. Blomberg and Daniela Frasca
}

University of Miami School of Medicine, Miami, Florida, USA.

\begin{abstract}
The burden of disease during seasonal influenza epidemics is felt most keenly among the very young and the elderly. Although vaccination effectively protects children and young adults against infection, it has limited efficacy in elderly individuals. This has been linked to a reduced ability to induce a robust serum antibody response. In this issue of the JCI, Sasaki et al. identify some of the cellular and molecular deficits that underlie the reduced serum antibody response induced by influenza vaccination in elderly individuals. Importantly, they show that it is the quantity of the response, and not its quality, that needs to be improved if we are to enhance the success of influenza vaccination in this vulnerable population.
\end{abstract}

Conflict of interest: The authors have declared that no conflict of interest exists.

Citation for this article: J Clin Invest. 2011;

121(8):2981-2983. doi:10.1172/JCI58406
Influenza epidemics are associated with an estimated 200,000 hospitalizations and 35,000 deaths each year in the United States alone (1). Most of these adversely affected individuals are young children or elderly individuals. Vaccination against influenza has been described to be effective in preventing infection in healthy children and young adults; however, many of the elderly individuals who receive the vaccine still contract the infection and have secondary complications that lead to hospitalization and/or disability $(2,3)$. Despite this, influenza vaccination is still universally recommended for elderly individuals, because it does reduce disease severity, but understanding the mechanism(s) that underlies the limited response of the elderly to influenza vaccines could help direct the development of more effective approaches. 